\title{
La médiation, moyen de prévenir la crise urbaine en France?
}

\section{Ghyslaine Thorion}

\section{(2) OpenEdition}

1 Journals

Édition électronique

URL : http://journals.openedition.org/communicationorganisation/318

DOI : 10.4000/communicationorganisation.318

ISSN : $1775-3546$

Éditeur

Presses universitaires de Bordeaux

Édition imprimée

Date de publication : 1 décembre 2007

Pagination : 80-92

ISSN : 1168-5549

\section{Référence électronique}

Ghyslaine Thorion, «La médiation, moyen de prévenir la crise urbaine en France ? », Communication et organisation [En ligne], 32 | 2007, mis en ligne le 01 décembre 2010, consulté le 30 avril 2019. URL: http://journals.openedition.org/communicationorganisation/318; DOI : 10.4000/

communicationorganisation.318 
Dossier : La ville dans tous les sens

Résumé

Afin de prévenir la crise urbaine et le malaise que connaissent les banlieues, des actions politiques et sociales ont été mises en oeuvre. L'enjeu des programmes politiques successifs (réhabilitation de l'habitat social, amélioration du cadre de vie) était de faire resurgir de la civilité et de la mixité sociale. Les manifestations violentes de banlieue des années 90 ont montré les limites de cette politique. Une nouvelle orientation est alors prise ; elle mise sur l'insertion par l'économique et le désenclavement des zones urbaines sensibles. Cette politique de rénovation urbaine a permis d'institutionnaliser la médiation comme mode de régulation sociale. D'initiative citoyenne, la médiation a été intégrée par les pouvoirs publics dans leurs politiques de la ville, comme gage de paix sociale. La pratique de la médiation prend des formes diverses, elle se veut à la fois pacificatrice, intégratrice et créatrice de lien social. Les premières évaluations territoriales de la médiation urbaine prouvent son utilité sociale.

\title{
Mots-clés
}

Crise urbaine, prévention, médiation sociale, médiation urbaine, politiques publiques, banlieues sensibles, communication de proximité, régulation sociale, pratiques de médiation sociale, utilité sociale.

\begin{abstract}
In order to prevent urban conflicts and trouble in outer suburbs, political and social urban policies have been implemented. What was at stake in successive political projects (rejuvenation of social housing and improvement of residents' local environment) was to give an impulse to citizenship and courtesy as well as mixed social relationship. The violent urban local conflicts of the 90's have shown the limit to such policies. Consequently, a new direction is been taken; it relies on the economic vector to stop the isolation and facilitate the integration of sensitive urban areas. This policy of urban rejuvenation has established mediation institutionally as a means of social regulation. As citizen initiative, mediation was integrated by governmental urban policy as a promise of social peace-building. Mediation implemented along different lines can be given different forms: pacification, social and racial integration and the creation of social interaction. The first observations of local practices in mediation have proved its social use.
\end{abstract}

\section{Key-Words}

Urban conflicts, prevention, social mediation, urban mediation, public urban policies, sensitive urban areas, local communication, regulation of social relationships, urban practices in mediation, social use.

Ghyslaine Thorion est Docteur en sciences de l'Information et de la Communication, chercheur au GREC/O, enseignante en Masters de médiation (université de Poitiers, IFOMENE-I.C.P). Consultante et formatrice en communication. 


\section{La médiation, moyen de prévenir la crise urbaine en France ? Ghyslaine Thorion}

gh.thorion@laposte.net

Faire un état des lieux de la "crise urbaine", c'est la mettre en parallèle avec la crise sociale que traverse la France depuis plusieurs décennies. Elle est révélatrice d'une crise économique (aux effets observables : chômage, exclusion, précarité), d'une crise morale (absence de projet fédérateur, angoisse face à l'avenir, crise des valeurs et des institutions chargées de les transmettre) et d'un délitement du lien social (marqué par l'individualisme, l'isolement et par une occupation différenciée des espaces selon l'appartenance à telle ou telle classe sociale ${ }^{1}$ ).

La crise urbaine révèle des processus de désorganisation sociale ${ }^{2}$ : incivilités, dégradation de biens publics et privés, délinquance, problèmes de voisinage, hostilité envers certaines ethnies ou entre groupes ethniques... Ce contexte entretient un sentiment d'insécurité dans la population, victime ou non d'agressions quotidiennes ou ponctuelles, en tout cas témoin de la détresse humaine et de l'exclusion. La crise urbaine met également en lumière une marginalisation territoriale de populations défavorisées ${ }^{3}$, éloignées des centres-villes, dans des zones enclavées, qualifiées aujourd'hui de "zones urbaines sensibles" (ZUS). Des indicateurs dits de "sensibilité" mesurent ce malaise urbain : taux de chômage global, des moins de 25 ans, échec scolaire, taux de "population étrangère", proportion de jeunes, de familles nombreuses, de familles mono-parentales, taux d'allocataires sociaux, de bénéficiaires du R.M.I., nombre de délits, d'outrage et de violence à agents de la force publique, d'incidents scolaires. Afin de gérer la crise urbaine et le malaise des banlieues, des actions sociales et politiques ont été mises en œuvre. Nous nous proposons de les étudier ci-après en mettant en avant l'apport de la médiation sociale - objet de notre étude - à travers ses enjeux, objectifs et évolution récente.

\section{Les politiques de la ville pour prévenir les crises urbaines}

Dans le début des années 1970, nous sommes encore en période de croissance économique et le premier remède utilisé se veut un

\footnotetext{
${ }^{1}$ Wyvekens, A., 2005.

${ }^{2}$ Stébé, J-M, 2005, p. 11.

${ }^{3}$ Delarue, J-M, 1991.
} 
accompagnement social. Il se traduit dans la mise en place d'équipements sociaux, socioculturels et le recrutement de travailleurs sociaux. Comme le souligne J.-M Stébé, cette politique joue un rôle de contrôle sur le terrain ${ }^{4}$ davantage que de prévention. Avec les premiers symptômes de la crise urbaine, les interventions qui suivent porteront d'abord sur la rénovation du cadre bâti, ensuite sur l'aménagement d'équipements économiques, tout en renforçant l'intervention sociale (animation sociale mais aussi lutte contre les pratiques déviantes). L'amélioration de l'habitat (mise aux normes de confort, d'isolation, réhabilitation d'ensembles HLM considérés comme vétustes) fait l'objet de deux programmes de revalorisation successifs :

- Habitat et vie sociale (HVS) entre 1973 et 1983 (une cinquantaine d'opérations est réalisée) qui porte sur le cadre bâti.

- Banlieue 89 entre 1983 et 1989 qui met en avant l'esthétique du bâti (400 interventions sont subventionnées par l'Etat).

«On pensait que l'architecture pouvait susciter de la civilité et, par-là, reconstituer le lien social là où il était censé avoir disparu », affirme Stébé. ${ }^{5}$ Il s'est avéré que l'amélioration architecturale, aussi nécessaire qu'elle soit, restait insuffisante à soigner le mal-être des populations dites défavorisées. Ces dispositifs de revalorisation fortement décriés ${ }^{6}$ n'intervenaient que sur le cadre physique sans prendre en compte le cadre de vie ni associer les habitants à la réflexion en amont de la réhabilitation. L'autre reproche fait à cette revalorisation était de se cantonner à la dimension d'un quartier, tendant à stigmatiser encore plus ses habitants. Les manifestations survenues au cours de l'été 1981 dans le quartier des Minguettes à Vénissieux ou celles qui ont suivi à l'automne 1990 dans les communes de Sartrouville, de Mantes-la-Jolie et de Vaulx-en-Velin, avec les incidents violents que l'on sait, ont alors marqué un tournant dans les politiques de la ville. Selon L.L. Chebbah-Malicet ${ }^{7}$, «il ressort de ces journées largement couvertes par les médias nationaux et locaux, une prise de conscience brutale d'un malaise lié à une urbanisation que l'on avait pensée en partie réussie et maîtrisée, et de l'existence d'une "jeunesse issue de l'immigration", catégorie jusque-là quasi-ignorée des pouvoirs publics ». La politique de la ville se trouve désormais axée sur la lutte contre l'exclusion et le développement social urbain. Chacun des

\footnotetext{
${ }^{4}$ Stébé, op.cit, p.11.

${ }^{5}$ Stébé, op.cit, pp. 12-13.

${ }^{6}$ Merlin, P., 1999, p. 83.

${ }^{7}$ Chabbah-Malicet, L-L, 1999.
} 
termes est important puisqu'il prend en compte d'une part le développement économique (à l'aide de dispositifs d'insertion professionnelle et de délocalisation des activités économiques), d'autre part la planification urbaine (avec une politique de l'habitat et d'aménagement des espaces publics). Cette politique de la ville passe également par des actions de prévention sociale.

Le programme porté en 1992, appelé «grand projet urbain » (GPU) associe à la démolition d'immeubles, fortement médiatisée, l'aménagement d'infrastructures routières et l'installation d'équipements publics afin de désenclaver l'habitat social. Des "contrats de ville" sont alors signés entre l'Etat, représenté par la DIV et les collectivités locales. Le programme qui suit, en 1999, se nomme "grand projet de ville" (GPV). L'échelle territoriale s'élargit du quartier à la ville, tout au moins sur le papier, car dans les faits, les lourdeurs politico-administratives ${ }^{9}$, le morcellement des responsabilités des différentes collectivités (dû à la décentralisation) ont souvent représenté un frein à la mise en place de ces programmes. C'est l'époque où l'on voit naître des "zones de redynamisation urbaine" (ZRU) avec les « zones franches urbaines (ZFU) à l'intérieur desquelles les entreprises, les artisans et les commerçants bénéficient d'aides financières et surtout d'exonérations fiscales lorsqu'ils créent des emplois $\gg{ }^{10}$. Les entreprises de ces zones, se trouvant à proximité de quartiers sensibles, ont pour objectif d'employer des jeunes exclus du marché du travail. Elles sont au nombre de 41 en 2004, de 100 en $2006^{11}$.

Enfin, instituée par la loi du 1er août 2003, dite loi d'orientation et de programmation pour la ville et la rénovation urbaine, la politique de "rénovation urbaine" remplace les GPV. Elle porte sur des programmes d'action dans des zones urbaines sensibles. La loi les définit en ces termes : « ces programmes d'action, qui tiennent compte du programme local de l'habitat, s'il existe, fixent, pour chaque zone et sur une période de cinq ans, des objectifs de résultats chiffrés relatifs à la réduction du chômage, au développement économique, à la diversification et à l'amélioration de l'habitat, à la restructuration ou à la réhabilitation des espaces et équipements collectifs, à la restructuration des espaces commerciaux, au renforcement des services publics, à l'amélioration de l'accès au système de santé

\footnotetext{
${ }^{8}$ DIV : Délégation Interministérielle à la Ville.

${ }^{9}$ Stébé, op.cit, p. 18.

${ }^{10}$ Stébé, op.cit, p. 19.

${ }^{11}$ Source : Délégation Interministérielle de la Ville.
} 
s'appuyant sur l'hôpital public, à l'amélioration du système d'éducation et de la formation professionnelle, de l'accompagnement social et au rétablissement de la tranquillité et de la sécurité publiques $\gg .{ }^{12}$ Comme nous le voyons, le champ des interventions de cette loi et des conventions qui s'y rattachent est large et se veut global en agissant sur divers plans (économique, social, urbain, environnemental, sécuritaire). Cette contractualisation met en présence à la fois l'Etat, les communautés d'agglomération, des partenaires privés. C'est le cas du Contrat Territorial de Rénovation Urbaine, signé en janvier 2007 par Plaine Commune ${ }^{13}$, qui intègre vingt-quatre projets de qualité de vie engagés sur les quartiers. Il recouvre une action globale de rénovation et de construction de logements, d'aménagement des espaces publics, d'insertion par l'emploi, d'amélioration des transports et de l'environnement. In fine, cette politique, présentée sur un axe de développement durable des territoires, est censée résoudre le mal-être des banlieues et favoriser la paix et la cohésion sociales. Dans cette dynamique de revitalisation urbaine, qu'en est-il de la dimension sociale et de la médiation?

\section{La médiation, une réponse à la crise des modes de régulation sociale}

Etymologiquement le terme "médiation" vient du latin mediare, être au milieu, s'interposer, être un intermédiaire entre deux êtres ou deux réalités. Espace "intermédiaire" de régulation sociale, la médiation nous vient des Etats-Unis. L'originalité du mouvement tient au fait qu'il est d'abord civique (fondé sur la solidarité et la communication dans la cité) et éthique (visant à responsabiliser la communauté et combler les lacunes politiques). Cette initiative citoyenne est apparue en France au milieu des années $70^{14}$. Le tiers secteur de la médiation est né sous l'impulsion soit de citoyens agissant individuellement ou au sein d'associations soit de professionnels ${ }^{15}$. Il coïncide d'une part avec le rejet de la norme et de toute forme d'autorité (y compris juridictionnelle), d'autre part avec la possibilité d'un recours à des solutions alternatives de résolution de conflits. Un sondage CSA

\footnotetext{
${ }^{12}$ Loi n 2003-710 du 1er août 2003.

${ }^{13}$ Plaine Commune (communauté d'agglomération de la banlieue nord de Paris) regroupe huit communes: Aubervilliers, La Courneuve, Epinay sur Seine, l'Ile St Denis, Villetaneuse, Pierrefitte sur Seine, Stains

${ }^{14}$ Briant, V. de, Palau, Y., 1999, pp. 120-121.

${ }^{15}$ tels des avocats à Lyon qui ont créé les premières "boutiques de droit" en 1975.
} 
réalisé en $2004^{16}$ laisse apparaître un manque de confiance des Français dans leur justice : l'égalité de tous devant la justice est remise en cause à $71 \%$, l'efficacité des peines face à la récidive dénoncée à $80 \%$. Par ailleurs, la justice apparaît trop lente $(87 \%)$, trop chère $(89 \%)$ et utilise un langage abscons $(81 \%)$. En revanche, la médiation comparativement plus rapide, moins onéreuse, permet une implication des intéressés dans la résolution de leurs différends et le maintien du lien social grâce à la présence d'un tiers, coordinateur des échanges, catalyseur et pacificateur. Ici pas de "mis en cause", pas de "plaignant" mais des médiés qui s'engagent librement dans un processus de communication et tenteront de négocier des relations nouvelles durables ou une solution équitable satisfaisante pour chacun d'eux. Les parquets peuvent ainsi être amenés à recourir à la médiation pour traiter des petites affaires (classées souvent sans suite): de délinquance quotidienne, d'infractions pénales (délit, contravention) ou de litiges d'ordre civil (voisinage, consommation, logement). Michèle Guillaume-Hofnung ${ }^{17}$ insiste sur la position du tiers dans la médiation : " la médiation se définit comme un mode de construction et de gestion de la vie sociale grâce à l'entremise d'un tiers, neutre, indépendant sans autre pouvoir que l'autorité que lui reconnaissent les médiés qui l'auront choisi ou reconnu librement $»$. Hubert Touzard ${ }^{18}$, pour sa part, définit la médiation comme un passage d'un état à un autre état: « la médiation se trouve parfois être la seule possibilité de rétablir une communication devenue impossible. Même si le résultat se limite à cela, la médiation a prouvé son utilité et son sens ». L'une et l'autre définition peuvent tout à fait s'appliquer à la médiation en milieu urbain. Il nous faut cependant chercher à comprendre la spécificité de la médiation urbaine ${ }^{19}$ par rapport aux divers modes d'intervention sociale. La médiation urbaine, mode local de régulation sociale, facilite le vivre-ensemble, permet la participation citoyenne dans l'expression des besoins, la recherche de solution consensuelle ou la conception de projets. Elle est un moteur de transformation sociale. Elle sert le maintien ou la création de liens sociaux, la prévention des conflits et le rétablissement du dialogue entre les publics.

\footnotetext{
${ }^{16}$ Sondage exclusif CSA / sélection du Reader's Digest mené sur le thème "Les Français et la Justice" et réalisé par téléphone le 29 juillet 2004 auprès d'un échantillon représentatif de la population française de 1002 personnes.

${ }^{17}$ Guillaume-Hofnung M., 1995, p. 74.

${ }^{18}$ Touzard, H. in Bonafé-Schmitt, J-P, 1999, p. 8 (Préface).

19 Nous définirons "la médiation urbaine" comme l'ensemble des pratiques de médiation sociale intervenant en milieu urbain. Notre acception se veut plus large que la médiation engagée par une communauté territoriale qui se nomme médiation urbaine.
} 
Les institutions publiques ${ }^{20}$ s'approprient à leur tour la médiation comme une alternative de régulation sociale. Cette médiation publique $^{21}$ s'est développée à la faveur d'une politique de la ville axée sur la prévention et l'insertion. Nous citerons ainsi les contrats locaux de sécurité (CLS) - dans le domaine de la prévention de la violence et de la délinquance -, les politiques en matière de logement des personnes défavorisées, celles prônant une justice de proximité - avec la création de Maisons de justice et du droit (MJD) et d'antennes juridiques et de médiation-, les projets d'urbanisme impliquant la participation des habitants ${ }^{22}$ et enfin les politiques publiques territoriales (notamment des communes ${ }^{23}$ ). La politique de la ville a évolué d'une politique d'équipement vers une logique de services (à travers le Développement Social des Quartiers, 1980) pour aboutir à une présence sociale au plus près des habitants (médiation urbaine, 1988). Les emplois de médiation urbaine sont en adéquation avec les besoins des ZUS dans les domaines de la prévention de la violence et de la délinquance, dans la sécurisation des usagers ou des habitants, dans la résolution des dysfonctionnements sociaux par le dialogue, dans la participation à la veille sociale. Les politiques de la ville misent également sur l'intégration des populations fragilisées par l'insertion professionnelle. Pour répondre à cet objectif, des dispositifs ont été mis en place. Ils se nomment régies de quartier, entreprises d'insertion, collectivités et services publics pour ce qui est des organisations offrant des emplois de ville ${ }^{24}$ ou PIMMS ${ }^{25}$, PSP $^{26}$ pour les services de proximité, et missions locales pour ce qui est de l'aide à l'insertion professionnelle et sociale des jeunes.

\footnotetext{
20 "A l'initiative des pouvoirs publics (Etat et collectivités territoriales), de grandes entreprises exerçant une activité publique (logement, transport...) et d'associations (...) dans le cadre des politiques de la ville". Stébé, op.cit, p. 78.

${ }^{21}$ Au sens où l'emploient Briant et Palau, op.cit, pour désigner une médiation légale dans laquelle le partenaire principal est la puissance publique. Versus "médiation privée"

${ }_{22}^{22}$ Dossier Ville et médiation.

${ }^{23}$ Dossier Médiation dans la ville.

24 emplois aidés, emplois-jeunes in Petitclerc, J-M, 2002, pp. 40-48.

25 "Les associations PIMMS (Point d'Information Médiation Multiservices ) sont nées de la volonté des entreprises de services publics de proposer des services de proximité adaptés aux personnes fragilisées et du besoin de reconstituer un lien social dans les quartiers sensibles (...) Les entreprise fondatrices, avec le soutien des pouvoirs publics, recherchent une solution mutualisée aux difficultés qu'elles rencontrent dans les quartiers". Source : DIV-CNFPT, 2004, p.94

${ }^{26}$ Les PSP (Points Services aux Particuliers) "fonctionnent sur le même principe que les PIMMS appliqués aux problèmes liés à la gestion des ressources familiales (surendettement, découverts bancaires récurrents, factures non réglées)".

Source : DIV-CNFPT, ibid, p.95
} 
Comme l'avance Jean-Pierre Bonafé-Schmitt ${ }^{27}$, nous pouvons craindre un risque d'instrumentalisation de cette médiation publique, tributaire des modèles et impératifs institutionnels et qui passe par la restitution d'informations aux services concernés (bailleurs, acteurs privés professionnels ou acteurs publics) et l'observation de besoins latents. Rien à voir avec la médiation citoyenne, issue de la société civile, appelée aussi médiation privée qui met en relation un tiers indépendant avec des personnes de statut privé qui s'engagent librement dans un processus de médiation. La médiation urbaine s'inscrit nécessairement dans une approche territoriale. Ce qui explique la diversité des structures de médiation que l'on rencontre sur le terrain tant sous l'aspect de leur fonctionnement, de leurs intervenants ou de leurs missions. Dans les faits, on assiste à une médiation de proximité fondée sur une communication relationnelle permanente, à adapter en fonction d'un contexte social mouvant.

\section{Les objectifs et les enjeux de la médiation urbaine}

- Etre à l'écoute des attentes de la population afin de donner une réponse à ses besoins, veiller à sa tranquillité. Les médiateurs sociaux qu'ils se nomment agents locaux de médiation sociale ${ }^{28}$, agents de prévention et de médiation dans les espaces publics ${ }^{29}$, correspondants de nuit ${ }^{30}$, agent d'ambiance dans les transports urbains $^{31}$ ou aides éducateurs ${ }^{32}$ agissent au plus près des publics

\footnotetext{
${ }^{27}$ Bonafé-Schmitt, J-P et al., 1999, p.35

${ }^{28}$ ALMS : leur mission est la prévention des incivilités et de la délinquance, la sécurité du cadre de vie, l'amélioration des relations sociales, la prévention et la résolution des conflits.

${ }^{29}$ Ils assurent une "veille technique et sociale, informe et oriente les habitants", contribuent à la "tranquillité des espaces publics et/ou ouverts au public". "A l'écoute des personnes ou des groupes, ils sont attentifs aux difficultés de la cohabitation résidentielle ou aux conflits d'usage des espaces et sont amenés à intervenir par le dialogue auprès des personnes ou des groupes pour réguler les tensions ou prévenir les situations à risques". Source : DIV-CNFPT, op.cit, p.57

${ }^{30}$ Médiateurs nocturnes, "ils contribuent à la qualité de la vie résidentielle nocturne et à la tranquillité des personnes par une présence humaine rassurante la nuit, et à la régulation de conflits d'usage des espaces, de manière à créer un climat de confiance, à prévenir les dégradations et à réduire les incivilités." "Fonction d'écoute et d'aide aux personnes en difficulté la nuit". Source : DIV-CNFPT, op.cit, p.73

Leur rôle est aussi celui de repérage des dégradations et de maintenance.

${ }^{31}$ Ils renforcent la présence humaine sur le réseau des transports, "assurent l'interface entre les usagers/clients et la société de transport". "Médiateurs travaillant en équipe, ils contribuent par le dialogue et l'information à la gestion de l'ambiance et à la qualité des services rendus aux clients-voyageurs dans les stations ou dans les vus, tramway, métro, train" Source : DIV-CNFPT, op.cit, p.64
} 
afin d'humaniser et de tranquilliser leurs espaces de vie, faire respecter ces espaces, les rassurer, comprendre leurs difficultés afin de les orienter. Une relation personnalisée d'écoute et d'assistance, une prévention des nuisances et de la délinquance. En un mot, une médiation pacificatrice, sécuritaire et une veille sociale.

- Créer ou réparer le lien social : le médiateur interculturel, qu'il s'agisse de femmes-relais ou d'adultes-relais ${ }^{33}$, favorise la communication entre habitants, entre générations, entre personnes de cultures différentes. Il impulse une démarche d'autonomie et de participation chez les personnes de différentes communautés, les accompagne dans leur démarche vers les structures sanitaires et les services publics. Nous pouvons qualifier cette médiation d'intégratrice, fondée sur une reconnaissance des identités culturelles.

- Participer à la régulation sociale. Le rôle d'interface du médiateur urbain lui permet de gérer les conflits par le dialogue, de réguler les tensions entre individus, entre groupe. Cette position de tiers neutre, impartial lui permet de se distancier par rapport à la situation, de favoriser l'interaction, l'intercompréhension et d'accompagner les intéressés dans la résolution de leur différend. Une médiation de régulation sociale reposant sur la recherche de consensus.

- Informer sur les droits, responsabiliser, éveiller à la citoyenneté. C'est ainsi la fonction des délégués au Médiateur de la République $^{34}$ ou des médiateurs sociaux en PIMMS. En fournissant des lieux de parole afin de traiter les problèmes au fur et à mesure qu'ils apparaissent, le médiateur accompagne, oriente. Tout repose sur un travail pédagogique d'explication.

\footnotetext{
32 «Affectés dans les établissements scolaires implantés sur une zone d'éducation prioritaire, ils ont un rôle à la fois de prévention des conduites déviantes, d'aide aux élèves en difficulté scolaire et sociale, d'accompagnement des jeunes exclus de leurs établissements, de médiation et de gestion des conflits ». Stébé, op.cit, p.21

33 «A partir d'initiative de femmes, souvent bénévoles au départ» (d'où le nom de femmes-relais), ces démarches citoyennes ont bénéficié d'une reconnaissance institutionnelle à travers les programmes d'adultes-relais. Souvent d'origine étrangère, ces femmes pratiquent l'accueil, l'accompagnement et l'orientation des personnes et familles du quartier, facilitent l'accès aux services publics, animent de nombreuses activités (fêtes de quartier, alphabétisation, ateliers d'expression...).

Source : Villes et médiation, op.cit, p. 12

${ }^{34}$ Installé dans les quartiers sensibles, il écoute et oriente les particuliers vers les administrations, recueille et traite les réclamations.
} 


\section{Une médiation d'intégration.}

Jean-François $\operatorname{Six}^{35}$ qualifierait cette médiation urbaine à la fois de "médiation créatrice" (parce qu'elle suscite des liens nouveaux), "rénovatrice" (elle réactive des liens distendus), "préventive" (dans le sens où elle prévient les conflits) et "curative" (lorsqu'elle aide les personnes ou groupes en conflit à en trouver la solution). Au regard des objectifs de la médiation urbaine et du cadre politico-historique qui a prévalu à son développement, nous pouvons clairement distinguer ses enjeux. Ils vont de la tranquillité urbaine à la cohésion sociale en passant par une veille sociale. Cette dernière s'appuie sur un diagnostic territorialisé des besoins sociaux, des réponses à y apporter localement en termes de médiation, d'amélioration de services mais aussi en termes de ressources partenariales (appel à d'autres acteurs du domaine social ou éducatif) et de réseaux à créer.

\section{L'ancrage de la médiation urbaine}

Comme on l'a vu, il existe une pluralité de pratiques de médiation. La prolifération de dispositifs dits de médiation, d'origine privée ou publique, et du terme même de "médiateur", parfois pour requalifier des métiers qui ne comportent qu'un volet médiation ${ }^{36}$, tend à décrédibiliser les fondements de la médiation et interroge la déontologie de la profession. Michèle Lindeperg, rapporteure pour le Conseil économique et social, souligne que «le cadre associatif est actuellement le facteur central de stabilisation des compétences, de professionnalisme, d'homogénéisation des pratiques $»^{37}$. En effet, les associations affiliées à un groupement professionnel ont mis en place un code de déontologie, des outils d'évaluation, des formations, des échanges sur leur pratique. Afin d'assurer la visibilité de la médiation en zone urbaine, une charte de référence de la médiation sociale a été établie en $2001^{38}$. Elle pose le cadre déontologique de l'intervention : « neutralité et impartialité, négociation et dialogue, libre consentement et participation des habitants, mobilisation des institutions, protection des droits des personnes, respect des droits fondateurs ». La charte reconnaît la diversité des pratiques de médiation sociale et le besoin de clarifier l'appellation "médiation sociale" en lui appliquant strictement la déontologie énoncée ci-avant. A l'évidence, il nous paraît utile de légitimer des interventions de médiation urbaine

\footnotetext{
${ }^{35}$ Six J-F, 1990, p. 164.

${ }^{36}$ Le gardien d'immeuble est devenu "médiateur technique".

${ }^{37}$ Lindeperg M., 2001, p.12.

${ }^{38}$ Document adopté par le groupe de travail interministériel et interpartenarial sur les emplois dits "de médiation sociale" DIV-CNFPT, op.cit, pp. 263-271.
} 
disparates en leur offrant un cadre et des règles de conduite précis. Cette charte est un premier pas. La relation ternaire institutionsmédiateur-habitants situe bien la médiation sociale dans le champ public. En revanche, la dénomination "employeur" pour désigner l'institution met à mal le principe d'indépendance du médiateur. Dans cette charte, quatre conditions pour l'exercice de la médiation sociale sont mises en avant: " les aptitudes du médiateur, la formation, l'encadrement et le partenariat». Est soulignée la nécessité d'une formation initiale et en alternance des médiateurs sociaux, identifiés sous leurs différentes appellations. Il est vrai que la formation a souvent fait défaut lors du recrutement des jeunes de banlieue dans cet emploi. Une professionnalisation assise sur une formation ${ }^{39}$ et sur un statut véritable ${ }^{40}$ permettra d'asseoir la légitimité de la pratique des médiateurs en zone urbaine. Bien entendu, dans ces métiers de contact, la dimension humaine joue un rôle indéniable. Elle passe par la confiance instaurée avec les acteurs et les qualités relationnelles dont fait preuve le médiateur sur le terrain.

L'ancrage de la médiation urbaine, c'est aussi une évaluation de son utilité sociale. Des enquêtes mettent en évidence la plus-value apportée par les médiateurs. Une corrélation a d'ailleurs été établie entre leur présence et l'amélioration des conditions de vie dans les quartiers. Elle met en avant les résultats suivants : «diminution du nombre des dégradations, augmentation des recettes dans les bus, augmentation du nombre de logements occupés dans un quartier populaire, baisse des demandes de changement de logement et de quartier, fréquentation de certains équipements sportifs ou culturels, baisse des réclamations des usagers $»^{41}$. Les démarches d'analyses sont bien entendu locales et ont chacune leurs indicateurs quantitatifs et qualitatifs. C'est le cas de l'étude menée en 2005 sur l'agglomération d'Angoulême ${ }^{42}$. Il apparaît cependant nécessaire d'homogénéiser ces diverses initiatives pour évaluer l'apport global de la médiation sociale. Un groupe de réflexion ${ }^{43}$ est né en janvier 2007. Il a pour

\footnotetext{
${ }^{39}$ Petitclerc, op.cit, p. 2 et p. 49.

${ }^{40}$ Il s'agit souvent d'emplois aidés, précaires.

${ }^{41}$ DIV-CNFPT, op.cit, p. 28.

${ }^{42}$ Le rapport d'enquête a été réalisé par Jacques SALZER, maître de conférence à l'université de Paris-Dauphine, co-créateur de formations à la médiation aux universités de Paris II, Paris V et au CNAM et Jean-Edouard GRESY, médiateur, chercheur en anthropologie à l'université de Paris I, consultant au Centre Européen de la Négociation.

${ }^{43}$ Il est constitué du groupement d'employeurs OMEGA d'Angoulême, l'ADEMN Médiation sociale de Lille, le GIP Chalon Sécurité Education Préventive de Chalon-
} 
objectif d'établir des outils d'évaluation de la médiation sociale qui seront ensuite diffusés au niveau national auprès des décideurs et responsables opérationnels. L'impact de la médiation urbaine pourra ainsi être estimé sur tout le territoire français. Cette évaluation permettra d'améliorer les dispositifs, de les réajuster, de structurer les coûts, de comparer les expériences dans ce domaine et d'établir d'autres indicateurs sociaux. Mesurer l'utilité sociale de la médiation urbaine ne pourra que donner du poids et de la valeur à un processus qui doit se pérenniser au regard des besoins. Néanmoins l'évaluation de la médiation urbaine ne pourra servir de baromètre pour mesurer le climat social. Il faut relativiser sa portée dans le sens où ce qui paraît utile socialement à un moment $\mathrm{T}$ ne l'est peut-être plus à un moment $\mathrm{T}+1$. La médiation sociale est en constante évolution au même titre que les attentes des populations et l'évolution des groupes de population. C'est d'ailleurs une des caractéristiques propres à la médiation urbaine : une approche pragmatique du terrain qui repose sur une proximité sociale. La régulation sociale pratiquée est souvent une réponse au cas par cas. Elle ne peut à elle seule soulager les causes structurelles de la crise, les choix politiques et les défaillances du service public. La médiation urbaine a besoin d'être soutenue par des politiques dynamiques qui prennent en compte les suggestions des médiateurs, les besoins de ces populations. Elle ne doit pas simplement pacifier ou apaiser mais avoir "une fonction d'alerte sociale" ${ }^{44}$, de remise en cause de l'existant si ce dernier s'avère peu efficace. A un niveau plus global, la médiation urbaine doit être une opportunité de réformes et de modernisation.

\section{Bibliographie}

Bonafé-Schmitt, J-P., Dahan J., Salzer J., Touzard H., Vouché, JP., Les médiations, la médiation, Ramonville, Editions Erès, 1999.

Briant V., de Palau Y., La médiation, Editions Nathan/HER, 1999.

Chebbah-Malicet, Laure-Leyla, «Des liaisons dangereuses? Intégrer les "immigrés" et gérer la crise urbaine », Ecarts d'identité, n ${ }^{\circ}$ 90/91 : Interculturalité et action publique, sept-déc. 1999.

Delarue J-M., Banlieues en difficulté : la relégation, Paris, Editions Syros-Alternatives, 1991.

sur Saône, l'Association de Médiation Sociale de Marseille et l'Agence Municipale de Médiation de Niort

44 "Ville et médiation", op.cit, p. 24. 
Dossier : La ville dans tous les sens

Délégation interministérielle à la ville (DIV) et Centre national de la fonction publique territoriale (CNFPT), La médiation sociale : une démarche de proximité au service de la cohésion sociale et de la tranquillité publique, Imprimerie Moderne de l'Est, 2004 (Repères).

Grésy J-E., Salzer J., Enquête de satisfaction auprès des habitants et des partenaires de la médiation sociale: évaluation du groupe d'employeurs O.ME.GA, DIV, juin 2005.

Guillaume-Hofnung M., La médiation, Paris, Editions PUF (Que sais-je ?), 1995.

Lindeperg M., Médiation et conciliation de proximité, rapport public présenté au nom de la section du cadre de vie, Conseil économique et social, La documentation française, 2001.

Merlin P., les banlieues, Paris, Editions PUF (Que sais-je ?), 1999.

Stébé J-M., La médiation dans les banlieues sensibles, Paris, PUF, 2005.

Six J-F., Le temps des médiateurs, Paris, Editions du Seuil, 1990.

Petitclerc, J-M., Pratiquer la médiation sociale : un nouveau métier de la ville au service du lien social, Paris, Editions Dunod (Collection : Action sociale), 2002.

Wyvekens A ., "La ville. Quelle crise urbaine ? Quelle politique?», in La documentation française, $\mathrm{n}^{\circ} 326$ : Comprendre la société, maijuin 2005 .

Dossier «La médiation dans la ville », Médiations et sociétés, 5 octobre 2003, pp. 11-27.

Dossier « Ville et médiation », dossier documentaire réalisé par Ferré, Isabelle (Le Cubitus), Porchet, Françoise (Centre de Documentation de l'Urbanisme), CDU, 2002.

Zones franches urbaines

http://www.ville.gouv.fr/pdf/editions/zfu-mode-emploi-2007.pdf 
